
Programa de Pós-Graduação em Engenharia de Produção - PPGEP
Laboratório de Qualidade de Vida - LaQVida
Universidade Tecnológica Federal do Paraná - UTFPR Ponta Grossa - PR - Brasil
v. 06, n. 04 , out./dez. 2014 , p. $232-240$
DOI: $10.3895 / \mathrm{S} 2175-08582014000400004$

\section{REVISTA BRASILEIRA DE QUALIDADE DE VIDA}

\title{
Qualidade de vida e independência funcional de usuários de drogas atendidos em um Centro de Atenção Psicossocial Álcool e Drogas (CAPSad)
}

\author{
Quality of life and functional independence of drug users treated at a \\ Psychosocial Care Center Alcohol and Drugs (CAPSad)
}

\author{
Lorena Cajaíba de Oliveira \\ Universidade Estadual do Sudoeste da Bahia - UESB - Jequié - Bahia - Brasil \\ lorenacajaiba@hotmail.com \\ Claudio Henrique Meira Mascarenhas \\ Universidade Estadual do Sudoeste da Bahia - UESB - Jequié - Bahia - Brasil \\ claudio12fisio@hotmail.com \\ Nayra dos Santos Andrade Melo \\ Universidade Estadual do Sudoeste da Bahia - UESB - Jequié - Bahia - Brasil \\ nayrafisio@yahoo.com.br
}

\section{RESUMO}

OBJETIVO: Avaliar a qualidade de vida e independência funcional de usuários de drogas em tratamento em um Centro de Atenção Psicossocial Álcool e Drogas (CAPSad).

MÉTODOS: Estudo transversal, com 21 sujeitos, de ambos os sexos, que atenderam aos critérios: idade igual ou superior a 18 anos; cadastrados e acompanhados pelo CAPSad; comparecer nos dias em que as entrevistas foram realizadas; apresentar-se capaz de se expressar oralmente; concordar em participar do estudo assinando o Termo de Consentimento Livre e Esclarecido (TCLE). A coleta de dados foi realizada através da aplicação de um questionário composto por três blocos de informações: entrevista compreendendo as variáveis sociodemográficas, informações gerais sobre a saúde e o consumo de drogas, aplicação do questionário de qualidade de vida - SF-36 e a Escala de Medida de Independência Funcional (MIF). Os dados foram analisados mediante abordagem descritiva, com uso de frequências absolutas e relativas, médias e desvio-padrão, a partir do programa Statistical Package for Social Sciences (SPSS versão 15.0).

RESULTADOS: Da amostra, 95,2\% $(\mathrm{n}=20)$ eram do sexo masculino, com média de idade de 44,6 $( \pm 10,1)$ anos. Constatou-se que o álcool era a substância mais consumida, com $73,7 \%$ ( $n=14)$ dependentes. Quanto à frequência de droga consumida semanalmente, verificou-se que o álcool era a substância consumida por mais dias da semana, com $81,8 \%(n=9)$ da amostra fazendo uso três dias ou mais por semana. Na análise dos diferentes domínios do SF-36, o maior comprometimento ocorreu nos domínios aspectos físicos, com média de 36,9 $( \pm 41,5)$ e aspectos emocionais com média de 42,9 ( \pm 43,6). Em relação aos aspectos da MIF avaliados no estudo, os resultados revelaram que $95,2 \%(\mathrm{n}=20)$ eram independentes funcionalmente.

CONCLUSÕES: O consumo de álcool, tabaco e outras drogas altera negativamente a qualidade de vida da amostra pesquisada, já a independência funcional manteve-se preservada.

PALAVRAS-CHAVE: Qualidade de vida. Alcoolismo. Tabagismo. 


\section{ABSTRACT}

OBJECTIVE: To assess the quality of life and functional independence of drug users in treatment at the Center for Psychosocial Attention Alcohol and Drugs (CAPSad).

METHODS: Cross-sectional study with 21 subjects of both sexes, who met the criteria: aged 18 years or over; registered and accompanied by CAPSad; to attend on days when the interviews were conducted; be able to express orally; agree to participate in the study by signing the consent form. Data collection was conducted by a questionnaire, applied individually, consisting of three blocks of information which included: sociodemographic variables, general information about health and drug use; application of the quality of life questionnaire - SF-36; and the Scale of the Functional Independence Measure (MIF). Data were analyzed through the descriptive approach, using absolute and relative frequencies, means and standard deviation from the Statistical Package for Social Sciences program (SPSS version 15.0).

RESULTS: From the sample, 95.2\% $(n=20)$ were male, with a mean age of $44.6( \pm 10.1)$ years. It found that alcohol was the most commonly used substance, with $73.7 \%(n=14)$ dependents. About the frequency of weekly drug used, it was found that the alcohol was the consumed substance by more days of the week, $81.8 \%(n=9)$ with a use for three days or more per week. In the analysis of the different domains of the SF-36, the greater involvement occurred in areas physical aspects, with an average of $36.9( \pm 41.5)$, and emotional aspects with an average of $42.9( \pm 43.6)$. Regarding aspects of MIF evaluated in the study, the results revealed that $95.2 \% \quad(n=20)$ were functionally independent.

CONCLUSIONS: The consumption of alcohol, tobacco and other drugs negatively affects the quality of life of the sample studied, since the functional independence remained preserved.

KEYWORDS: Quality of life. Alcoholism. Smoking.

\section{Introdução}

O abuso ou dependência de álcool, tabaco e outras drogas, mais que um dilema das pessoas que fazem o seu uso, consiste num grave problema de saúde pública (GOMES, 2011). Essa problemática pode produzir condições médicas agudas e crônicas de difícil resolução, além de causar efeitos psicológicos, sociais, culturais, jurídicos, políticos e econômicos, gerando incapacidade junto às populações e até mortalidade (GIGLIOTTI; BESSA, 2004; LUIS; LUNETTA, 2005; ALIANE; LOURENÇO; RONZANI, 2006; MACHADO; KLEIN, 2007; SOARES, 2009).

De acordo com o United Nations Office on Drug and Crime (UNODC, 2013), houve aumento na produção e no uso de novas substâncias psicoativas mundialmente. Assim, faz-se necessária uma abordagem equilibrada para lidar com a problemática das drogas, intensificando medidas de prevenção e de tratamento, uma vez que o consumo indiscriminado continua comprometendo a saúde e o bem-estar de pessoas. Logo, a existência de centros de atendimento específicos torna-se fundamental para o desenvolvimento de estratégias de combate a essas substâncias (BARBANTI, 2006).

O Ministério da Saúde, em 2002, regulamentou o atendimento de pacientes com transtornos causados pelo uso prejudicial e/ou dependência de álcool e de outras drogas, em Centros de Atenção Psicossocial (CAPS). Os CAPS são unidades voltadas para o atendimento da saúde mental, no qual é oferecido o tratamento para pessoas com transtornos mentais, psicoses, neuroses graves e demais acometimentos que justifiquem o cuidado intensivo, comunitário, personalizado e promotor de vida. 
Essa instituição deve possuir espaço próprio com consultórios, salas para atividades em grupo, espaço de convivência, oficinas, refeitório, sanitários e área externa para recreação. Contudo, os CAPS não são iguais, sendo divididos da seguinte forma: CAPS I e CAPS II, para atendimento diário a adultos com transtornos mentais severos; CAPS III, com atendimento diário e noturno para transtornos mentais persistentes; CAPSi, para infância e adolescência; e, CAPSad, para usuários de álcool e drogas. Sua equipe deve ser composta por um médico psiquiatra, um enfermeiro com formação em saúde mental, um médico clínico, quatro profissionais de nível superior que podem ser: psicólogo, assistente social, enfermeiro, terapeuta ocupacional, pedagogo, e seis profissionais de nível médio, técnico e/ou auxiliar de enfermagem, técnico administrativo, técnico educacional e artesão (BRASIL, 2004; MONTEIRO et al., 2011).

Para Patrício (1995), o dependente de drogas é doente do ponto de vista médico e social. Esse doente apresenta, em alguns casos, retardo ou hiperatividade psicomotora, déficit de ação social e familiar, redução dos cuidados consigo mesmo e padrões de sono alterados, o que contribui para o comprometimento da qualidade de vida. Com efeito, o tratamento precisa de abordagem específica e diagnóstica, com cuidados gerais em saúde que compreendem serviço hospitalar, ambulatorial e grupos de autoajuda (BRASIL, 2004; PILLON; LUIS, 2004; MIRANDA et al., 2006; MONTEIRO et al., 2011; MARTINS et al., 2012).

A Organização Mundial de Saúde, através do The WHOQOL Group (1995), definiu qualidade de vida como a percepção que o sujeito tem sobre sua disposição na vida, levando em consideração sua cultura e valores que vão atuar intimamente na construção de objetivos, expectativas, padrões e preocupações. Conceito este influenciado pela saúde física, estado psicológico, relações sociais, nível de independência e relações mais relevantes. Sendo assim, a qualidade de vida é um parâmetro que pode ser utilizado em diversas áreas. No caso de usuários de drogas, com o parâmetro, é possível identificar a percepção que o indivíduo possui acerca de sua saúde e da doença que o acomete, auxiliando na tomada de decisões sobre o tratamento (BRITO et al., 2008; GOMES, 2011).

A saúde física ou o declínio da mesma interfere intimamente na percepção do indivíduo sobre sua qualidade de vida. Assim, é de extrema importância avaliar a independência funcional, uma vez que este marcador permite caracterizar a capacidade do indivíduo em realizar algo com os próprios meios e, para isso, supõem-se condições motoras e cognitivas satisfatórias (RIBERTO et al., 2004).

Diante do impacto causado pelo abuso/dependência de substâncias psicoativas, percebe-se que existe alteração na qualidade de vida e independência funcional dos indivíduos, uma vez que suas funções físicas, mentais, intelectuais, emocionais e o convívio com outras pessoas em diversos ambientes se tornam prejudicadas. Embora a evolução da independência física e cognitiva faça parte do processo de reabilitação, pouco se tem estudado, mesmo sendo estes marcadores importantes para obtenção de resultados na área de saúde (ESCUDEIRO et al., 2006; BARBANTI, 2006). Sendo assim, o presente estudo objetivou avaliar a qualidade de vida e independência funcional de usuários de drogas em tratamento em um Centro de Atenção Psicossocial Álcool e Drogas (CAPSad).

\section{Métodos}

Trata-se de um estudo transversal, realizado no mês de janeiro de 2014, com indivíduos de ambos os sexos, com histórico de uso de substâncias psicoativas (álcool, tabaco e outras drogas), que buscaram tratamento no CAPSad, no município de Jequié, interior da Bahia.

Para inclusão dos participantes no estudo, adotaram-se como critérios: pacientes de ambos os sexos; com idade igual ou superior a 18 anos; que estavam cadastrados e sendo tratados no CAPSad estudado; ter comparecido nos dias em que as entrevistas foram realizadas; apresentaramse conscientes, orientados e capazes de se expressar oralmente; e, concordaram em participar do estudo assinando o Termo de Consentimento Livre e Esclarecido (TCLE). 
Para a realização do estudo foi utilizado um questionário composto por três blocos de informações, sendo aplicado na forma de entrevista, realizada pela investigadora principal e por um entrevistador previamente treinado. $\mathrm{O}$ primeiro bloco foi composto por variáveis sociodemográficas, informações gerais sobre a saúde e sobre o consumo de drogas.

O segundo bloco consistiu da avaliação da qualidade de vida, através da utilização do questionário SF-36, validado no Brasil por Ciconelli (1997). Esse instrumento é genérico, de fácil compreensão e rápida aplicação, dividido em oito domínios: capacidade funcional, aspectos físicos, dor, estado geral de saúde, vitalidade, aspectos sociais, aspectos emocionais e saúde mental. Para cada pergunta há somente uma resposta, sendo que, posteriormente, os itens são codificados e transformados no resultado final que é apresentado num escore de 0 a 100, no qual baixos escores correspondem a pior qualidade de vida e altos escores melhor qualidade de vida.

O terceiro bloco avaliou o desempenho da pessoa nas esferas motora e cognitiva, com o uso da Escala de Medida de Independência Funcional (MIF), desenvolvida na América do Norte em 1986, e que avalia as seguintes atividades: autocuidados, transferências, locomoção, controle esfincteriano, comunicação e cognição social. Cada item é pontuado de 1 a 7 , com um escore total variando de 18 a 126 pontos, sendo os sujeitos da pesquisa classificados da seguinte maneira: 0 a 18 pontos, dependência total; 19 a 60 pontos, dependência de 50,0\%; 61 a 103 pontos, dependência de 25,0\%; e, 104 a 126 pontos, independência (CICONELLI et al., 1999; PAYÁ et al., 2002; RIBERTO et al., 2004; RICCI; KUBOTA; CORDEIRO, 2005; BRITO et al., 2008; SILVA et al., 2012).

Os dados foram armazenados e analisados no programa Statistical Package for Social Sciences (SPSS) - versão 15.0, sendo a análise realizada mediante abordagem descritiva, com determinação de médias, desvio-padrão e frequências absoluta e relativa.

A pesquisa seguiu as normas éticas da Resolução n. 466/12 do Conselho Nacional de Saúde e foi aprovada pelo Comitê de Ética em Pesquisa da Universidade Estadual do Sudoeste da Bahia (UESB), com o parecer $n^{\circ} 475.592 / 13$, de 02 de dezembro de 2013. Para todos os participantes foram oferecidas informações sobre o procedimento da pesquisa e seus objetivos, e os mesmos aceitaram participar voluntariamente do estudo e assinaram o TCLE em duas vias, uma ficando com o sujeito.

\section{Resultados}

Foram abordados 26 pacientes que compareceram ao CAPSad no período das entrevistas. O processo foi finalizado quando se percebeu que todos os indivíduos já tinham sido abordados. Dos 26 sujeitos, 4 se recusaram a assinar o TCLE e 1 estava fora da faixa etária estabelecida, por conta disso, 21 pacientes participaram do estudo.

Dos 21 indivíduos entrevistados, 95,2\% $(\mathrm{n}=20)$ eram do gênero masculino, com média de idade de 44,6 $( \pm 10,1)$ anos, sendo 66,7\% $(n=14)$ trabalhadores autônomos (Tabela 1).

Tabela 1 - Valores referentes ao gênero, idade e tipo de serviço exercido pelos indivíduos investigados

\begin{tabular}{ccccccc}
\hline \multicolumn{2}{c}{ Gênero } & \multicolumn{2}{c}{ Idade } & \multicolumn{3}{c}{ Serviço Exercido } \\
\hline Masculino & Feminino & Valor mín. & Valor máx. & Servidor público & Autônomo & Desempregado \\
20 & 1 & 27 & 62 & 3 & 14 & 4 \\
$95,2 \%$ & $4,8 \%$ & Média: 44,6 & $14,3 \%$ & $66,7 \%$ & $19,0 \%$ \\
\hline \multicolumn{4}{c}{ Fonte: Autoria própria (2014). }
\end{tabular}

No que se refere ao tipo de droga em uso, constatou-se que o álcool era a substância mais consumida, com 66,7\% ( $\mathrm{n}=14)$ dos dependentes. Quanto à frequência de droga consumida, semanalmente, o álcool também obteve o maior resultado com 64,3\% ( $n=9)$ do total da amostra, tendo uso em três dias ou mais por semana (Tabela 2). 
Tabela 2 - Valores referentes ao tipo e quantidade de droga consumida pelos indivíduos investigados

\begin{tabular}{lcccccc}
\hline Tipo & Sim & Não & Omissões & Consumo 2X & Consumo 3X ou mais & Omissões \\
\hline Bebida alcoólica & $14(66,7 \%)$ & $5(23,8 \%)$ & $2(9,5 \%)$ & $2(14,3 \%)$ & $9(64,3 \%)$ & $3(21,4 \%)$ \\
Tabaco & $8(38,1 \%)$ & $11(52,4 \%)$ & $2(9,5 \%)$ & $0(0,0 \%)$ & $8(100,0 \%)$ & $0(0,0 \%)$ \\
Drogas ilícitas & $3(14,3 \%)$ & $16(76,2 \%)$ & $2(9,5 \%)$ & $0(0,0 \%)$ & $3(100,0 \%)$ & $0(0,0 \%)$ \\
\hline
\end{tabular}

Fonte: Autoria própria (2014).

$\mathrm{Na}$ análise dos diferentes domínios do SF-36, a maioria apresentou valores reduzidos, sendo as variáveis mais afetadas aspectos físicos $(36,9)$ e aspectos emocionais $(42,9)$, considerando-se os valores da média (Gráfico 1).

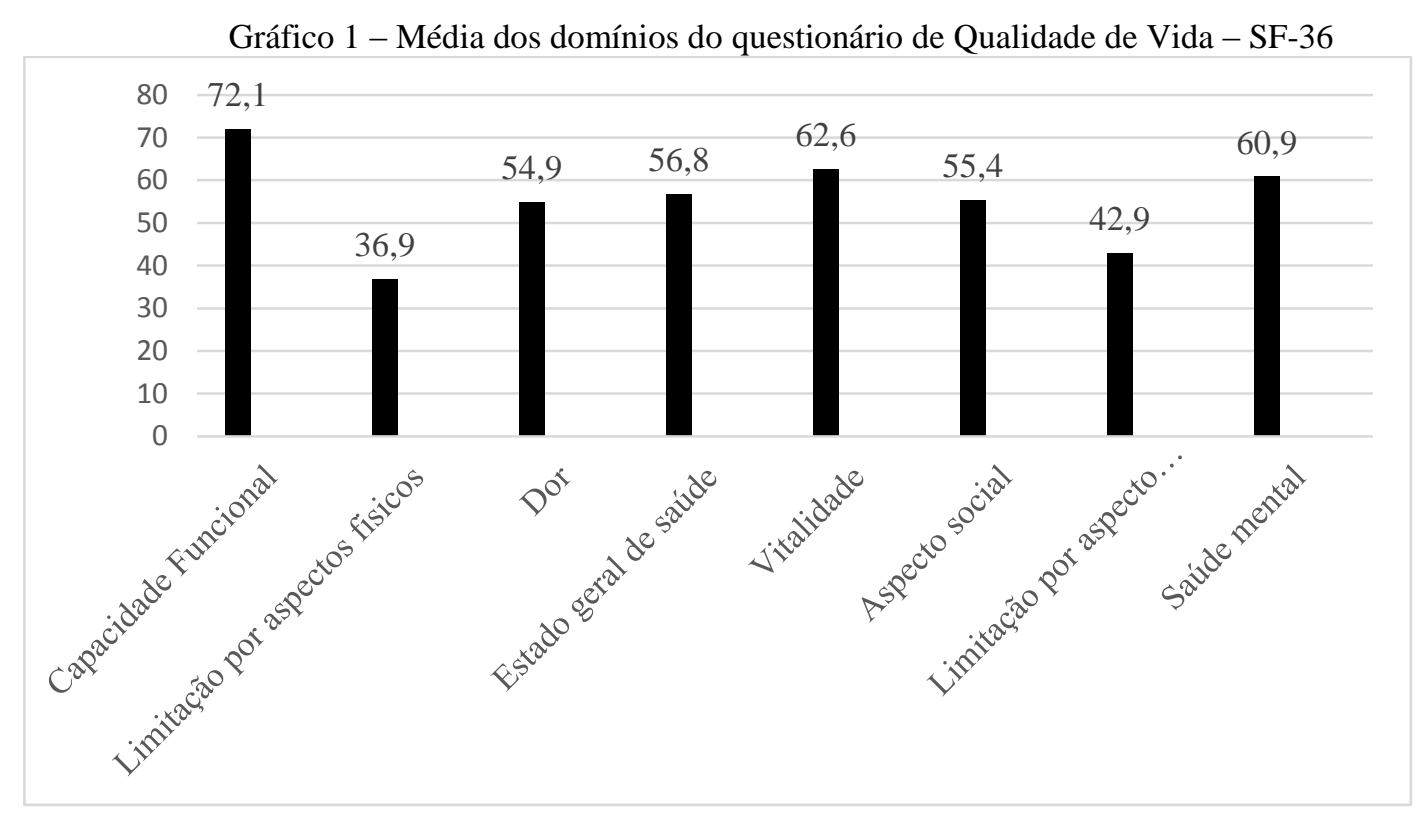

Fonte: Autoria própria (2014).

Em relação aos aspectos da MIF avaliados no estudo, os resultados revelaram que 95,2\% $(n=20)$ da amostra são independentes funcionalmente e apenas 4,8\% (n=1) possuía dependência parcial de $50,0 \%$.

\section{Discussão}

O conjunto de informações obtidas com o presente estudo mostrou que os usuários de drogas apresentaram comprometimento na maioria dos domínios da qualidade de vida. Esses resultados estão de acordo com o estudo de Büchele, Coelho e Lindner (2009), o qual diz que o uso de drogas atua como um agressor na vida moderna. Mas o problema pode ir além, pois se sabe que o seu consumo está em ascendência, e percebe-se que há prioridade quando se trata da repressão ao uso de drogas ilícitas, enquanto o combate às drogas lícitas é quase inexistente. Porém, vale ressaltar que o comprometimento físico, mental, emocional, profissional, familiar e social independe da legalidade do uso ou não. Qualquer consumo abusivo e indiscriminado traz prejuízos que interferem gravemente na qualidade de vida e independência funcional dos usuários. Segundo Luis e Lunetta (2005), as consequências das drogas são poderosos determinantes para a morbimortalidade.

Para Castro et al. (2007), a qualidade de vida é um marcador que vem ganhando importância como uma forma de avaliar o impacto causado por uma patologia e a eficácia da terapêutica proposta e adotada pelo indivíduo. Logo, a avaliação desse marcador é um importante critério para verificar o tratamento de cronicidades, como o abuso ou dependência de álcool, tabaco e outras drogas, permitindo assim, avaliar os diferentes domínios e direcionar as estratégias para objetivos mais precisos. 
O presente estudo identificou o comprometimento da qualidade de vida em diferentes domínios, levando a crer que o uso abusivo ou dependência das drogas prejudica a saúde física, mental, social e emocional dos indivíduos. Esse marcador pode indicar a situação na qual o indivíduo chegou à instituição e o impacto que o consumo abusivo lhe causou até aquele dado momento, a partir daí, observar como o paciente se comporta e adere ao tratamento e, por fim, discriminar quais foram os resultados obtidos, conferindo um feedback bastante específico tanto para a equipe de saúde, quanto para o paciente (MARTINS et al., 2012).

Os dados supracitados estão de acordo com estudos que avaliaram a qualidade de vida em pacientes usuários de álcool e tabaco com o instrumento SF-36, que têm comprovado que a qualidade de vida está diminuída em relação aos indivíduos não usuários de drogas. Payá et al. (2002), em estudo com indivíduos do sexo masculino dependentes de álcool, observaram uma qualidade de vida mediana, considerando-se as médias e os valores preconizados para o melhor padrão de qualidade de vida. Volk et al. (1997) também constataram que pacientes dependentes do álcool apresentam piores escores de qualidade de vida. Já Wilson, Parsons e Wakefield (1999), conduziram um estudo sobre o tabaco, o qual demonstrou prejuízos em todas as dimensões do SF36 entre os tabagistas moderados e graves. Castro et al. (2007), em pesquisa com tabagistas, concluíram que os fumantes obtiveram menor qualidade de vida quando comparado aos exfumantes e aos que nunca fumaram.

Segundo Dodgen e Shea (2000), o uso inconsequente de substâncias psicoativas demonstra desprezo pela saúde mental e física do sujeito, que evolui para deterioração do corpo, da mente e da vida social do indivíduo. Esta condição fica clara ao atentar-se para os resultados encontrados no presente estudo: aspecto físico $(36,9)$, aspecto emocional $(42,9)$ e aspecto social $(55,4)$, todos apresentando escores reduzidos.

A limitação por aspecto físico está relacionada com fatores como o tempo dedicado ao trabalho, realização de atividades aprazíveis, limitação no trabalho e dificuldades para realizar alguma atividade. Sua média bastante reduzida demonstra que, independentemente da idade ou do tempo de consumo, os pacientes possuem comprometimentos físicos, alguns, resultantes de quedas, acidentes e desavenças durante o consumo, que, consequentemente, levaram a dificuldade para desenvolver atividades cotidianas (BARBANTI, 2006; MACHADO; KLEIN, 2007).

$\mathrm{O}$ aspecto emocional avalia o tempo dedicado ao trabalho e à realização de atividades prazerosas, sendo que seu prejuízo pode indicar situação de sensação desagradável, levando o indivíduo a considerar-se não saudável. Para Gomes (2011), o uso de drogas age nas emoções e atua nos conflitos externos e internos, sendo o sofrimento psíquico um grande indicador de gravidade da dependência da droga. Logo, os resultados encontrados sugerem que o estado emocional está interferindo negativamente na condição dos indivíduos, sendo que os mesmos já não conseguem se dedicar ao trabalho, tendo assim que recorrer aos mais diversos meios para conseguir a obtenção da droga, o que traz à tona a questão social que envolve a temática em questão (BARBANTI, 2006).

$\mathrm{O}$ aspecto social é caracterizado pela interferência ou não da saúde física e problemas emocionais em atividades sociais, além do tempo dedicado a elas. Segundo Brito et al. (2008), pacientes com cronicidades limitam seu tempo às atividades cotidianas, que podem culminar na redução ou exclusão do convívio com familiares, trazendo prejuízos para os relacionamentos sociais e para as atividades prazerosas. Portanto, a qualidade emocional e social devem ser preconizadas no tratamento e reabilitação dos adictos (BARBANTI, 2006; MACHADO; KLEIN, 2007; BRITO et al., 2008).

A avaliação da capacidade funcional faz referência à presença e à extensão de limitações relacionadas à prática de atividades físicas que exigem pequeno, médio e grande esforço. A média obtida no presente estudo foi a maior entre os domínios avaliados $(72,1)$, sugerindo assim que o abuso/dependência de álcool, tabaco e outras drogas não trouxeram tantos prejuízos como outras doenças crônicas.

$\mathrm{Na}$ avaliação do domínio dor, verificou-se a presença de sofrimento, baseado na intensidade, extensão e interferência de dor na realização de atividades do cotidiano (BRITO et al., 2008). A média de 54,9 obtida nesse domínio demonstra que esta variável interfere na qualidade de vida dos 
pacientes entrevistados, e que apesar da dependência/abuso de substâncias manifestarem de forma específica em cada indivíduo, a dor, neste estudo, é uma sensação bem comum. Contudo, o estudo de Barbanti (2006), com pacientes dependentes químicos e depressivos, observou que os dependentes de drogas aparentaram sofrer menos dor que os indivíduos não dependentes.

O estado geral de saúde avalia a percepção do indivíduo sobre seu estado de saúde-doença, sendo que no presente estudo, a média desse domínio foi de 56,8, apresentando assim, prejuízo importante, fato que demonstra que os indivíduos não definem seu estado de saúde e sua evolução como bons. Situação que pode ser justificada pelo desconhecimento ou desinteresse da gravidade da situação/doença e da necessidade de tratamento contínuo. Esses resultados corroboram com os da pesquisa de Brito et al. (2008), realizada com doentes crônicos, que demonstrou importante redução do estado geral de saúde em comparação aos demais domínios, permitindo sugerir que cronicidades atuam comprometendo negativa e significativamente no estado geral de saúde.

A vitalidade reporta-se ao nível de energia e fadiga. $O$ valor médio obtido nesse domínio $(62,6)$ sugere que o uso de álcool, tabaco e outras drogas interferem na disposição do usuário do presente estudo. Logo, percebe-se a importância da prática de exercícios físicos como parte do tratamento para estes sujeitos, uma vez que seu efeito melhora o estado de humor, aumenta o nível de energia percebida pelos indivíduos, atuando sobre sua autoestima e promovendo melhora das relações afetuosas (FONSECA et al., 2000; BRITO et al., 2008).

A saúde mental está ligada às sensações como nervosismo, depressão, tranquilidade e felicidade (BARBANTI, 2006). Investigá-la é importante, uma vez que o uso de substâncias está relacionado às altas taxas de comorbidade por desordens mentais (MACHADO; KLEIN, 2007). No presente estudo, a média atribuída à saúde mental foi de 60,9 , o que demonstra um comprometimento neste item. Para Trefaut (1997), entre homens, o alcoolismo é o principal problema de saúde mental.

As limitações físicas interferem, certamente, na qualidade de vida de qualquer ser humano. Porém, os resultados do presente estudo indicaram que 95,2\% da amostra é independente funcionalmente, o que demonstra que apesar da qualidade de vida apresentar-se diminuída, principalmente nos aspectos físicos, a mesma não interferiu na capacidade do indivíduo de realizar autocuidados.

Os achados deste estudo, juntamente com a literatura, permitem inferir que o consumo de álcool, tabaco e outras drogas altera negativamente a qualidade de vida de seus usuários; já a independência funcional, nos sujeitos abordados, manteve-se preservada. Contudo, no decorrer da pesquisa, algumas limitações foram encontradas, destacando-se o tamanho da amostra e, também, a possível omissão de informações por parte dos entrevistados, uma vez que se trata do consumo e acesso às drogas lícitas e ilícitas.

\section{Referências}

ALIANE, P. P.; LOURENÇO, L. M.; RONZANI, T. M. Estudo comparativo das habilidades sociais de dependentes e não dependentes de álcool. Revista Psicologia em Estudo, v. 11, n. 1, p. 83-88, jan./abr. 2006.

BARBANTI, E. J. Efeito da atividade física na qualidade de vida em pacientes com depressão e dependência química. Revista Brasileira de Atividade Física e Saúde, v. 11, n. 1, p. 37-45, 2006.

BRASIL. Ministério da Saúde. Secretaria de Atenção à Saúde. Departamento de Ações Programáticas Estratégicas. Saúde mental no SUS: os Centros de Atenção Psicossocial. Brasília DF, 2004.

BRITO, M. S.; ARAÚJO, T. L.; GALVÃO, M. T. G.; MOREIRA, T. M. M.; LOPES, M. V. O. Qualidade de vida e percepção da doença entre portadores de hipertensão arterial. Caderno de Saúde Pública, Rio de Janeiro, v. 24, n. 4, p. 933-940, abr. 2008. 
BÜCHELE, F.; COELHO, E. B. S.; LINDNER, S. R. A promoção da saúde enquanto estratégia de prevenção ao uso de drogas. Revista Ciência e Saúde Coletiva, v. 14, n. 1, p. 267-273, 2009. crossef

CASTRO, M. da G.; OLIVEIRA, M. de S.; MORAES, J. F. D.; MIGUEL, A. C.; ARAÚJO, R. B. Qualidade de vida e gravidade da dependência do tabaco. Revista Psiquiatria Clinica v. 34, n. 2, p. 61-67, 2007. crossef

CICONELLI, R. M.; FERRAZ, M. B.; SANTOS, W.; MEINÃO, I.; QUARESMA, M. R. Tradução para a língua portuguesa e validação do questionário genérico de avaliação de qualidade de vida SF36 (Brasil SF-36). Revista Brasileira de Reumatologia, v. 39, n. 3, p. 143-150, maio/jun. 1999.

CICONELLI, R. M. Tradução para o português e validação do questionário genérico de avaliação de qualidade de vida Medical Outcomes Study 36-Item Short-form Health Survey (SF-36). 1997. 143 f. Tese (Doutorado em Ciências da Saúde Aplicadas à Reumatologia) Programa de Pós-Graduação em Ciências da Saúde Aplicadas à Reumatologia, Universidade Federal de São Paulo, São Paulo.

DODGEN, C. E.; SHEA, W. M. Substance use disorders - assessment and treatment. New York: Academic Press, 2000.

ESCUDEIRO, R.; LAMACHÃ, S.; FREITAS, A.; SILVA, H. Qualidade de vida e toxicodependência. Revista Toxicodependências, v. 12, n. 3, p. 65-78, 2006.

FONSECA, P. G. P.; TRAFANIUC, L.; TAVARES JÚNIOR, H.; MESQUITA, R. M.; LOTUFO NETO, F. Alterações nos estados de humor em pacientes com episódio depressivo grave após uma única sessão de atividade física. Jornal Brasileiro de Psiquiatria, Rio de Janeiro, v. 49, n. 6, p. 203-206, 2000.

GIGLIOTTI, A.; BESSA, M. A. Síndrome de dependência do álcool: critérios diagnósticos. Revista Brasileira de Psiquiatria, v. 26, p. 11-13, 2004. Suplemento 1.

GOMES, D. F. Qualidade de Vida, psicopatologia e programas terapêuticos na toxicodependência: um estudo realizado no CRI de Braga. 2011. 128 p. Dissertação (Mestrado em Psicologia Clínica e Saúde) - Faculdade de Filosofia, Universidade Católica Portuguesa, Braga, 2011.

LUIS, M. A. V.; LUNETTA, A. C. F. Álcool e outras drogas: levantamento preliminar sobre a pesquisa produzida no Brasil pela enfermagem. Revista Latino-americana de Enfermagem, v. 13, p. 1219-1230, nov./dez. 2005. rossef

MACHADO, P. P. P.; KLEIN, J. M. Drug dependence and psychological distress in Portuguese patients entering a substance abuse treatment center. International Journal of Clinical and Health Psychology, v. 7, n. 1, p. 83-91, 2007.

MARTINS, M. E.; RIBEIRO, L. C.; BARACHO, R. A.; FEITAL, T. J.; RIBEIRO, M. S. Qualidade de vida e consumo de alcoólicos em hepatopatas do sexo masculino. Revista Psiquiatria Clinica, v. 39, n. 1, p. 5-11, 2012. Crossef 
MIRANDA, F. A. N.; SIMPSON, C. A.; AZEVEDO, D. M.; COSTA, S. S. O impacto negativo dos transtornos do uso e abuso do álcool na convivência familiar. Revista Eletrônica de Enfermagem, v. 8, n. 2, p. 222-232, 2006.

MONTEIRO, C. F. S.; FÉ, L. C. M.; MOREIRA, M. A. C.; ALBUQUERQUE, I. E. M.; SILVA, M. G.; PASSAMANI, M. C. Perfil sociodemográfico e adesão ao tratamento de dependentes de álcool em CAPS-AD do Piauí. Escola Ana Nery Revista de Enfermagem, v. 15, n. 1, p. 90-95, jan./mar. 2011. Grossef

PATRÍCIO, L. D. Droga de vida, vidas de droga. Venda Nova: Bertrand, 1995.

PAYÁ, R.; FIGLIE, N. B.; TURISCO, J. L.; LARANJEIRA, R. Como é a qualidade de vida dos dependentes do álcool? Jornal Brasileiro de Psiquiatria, v. 51, n. 1, p. 39-45, fev. 2002.

PILLON, S. C.; LUIS, M. A. V. Modelos explicativos para o uso de álcool e drogas e a prática da enfermagem. Revista Latino-americana de Enfermagem, v. 12, n. 4, p. 676-682, jul./ago. 2004.

RIBERTO, M.; MIYAZAKI, M. H.; JUCÁ, S. S. H.; SAKAMOTO, H.; PINTO, P. P. N.; BATTISTELLA, L. R. Validação da versão brasileira da Medida de Independência Funcional. Revista Acta Fisiátrica, v. 11, n. 2, p. 72-76, 2004.

RICCI, N. A.; KUBOTA, M. T.; CORDEIRO, R. C. Concordância de observações sobre a capacidade funcional de idosos em assistência domiciliar. Revista Saúde Pública, v. 39, n. 4, p. 655-662, 2005. crossef

SILVA, G. A. da; SCHOELLER, S. D.; GELBKE, F. L.; CARVALHO, Z. M. F. de; SILVA, E. M. de J. P. da S. Avaliação funcional de pessoas com lesão medular: utilização da escala de independência funcional - MIF. Revista Texto e Contexto Enfermagem, Florianópolis, v. 21, n. 4, p. 929-936, out./dez. 2012.

SOARES, A. Variáveis psicossociais e reatividade emocional em cuidadores de dependentes de substâncias. 2009. 396 p. Tese (Doutorado em Psicologia da Saúde) - Universidade do Minho, Braga, 2009.

THE WHOQOL GROUP. The World Health Organization quality of life assessment (WHOQOL): position paper from the World Health Organization. Social Science and Medicine, v. 41, p. 1403-1410, 1995. rossef

TREFAUT, M. P. Dura volta à superfície. Carta capital, São Paulo, n. 45, p. 74-77, abr. 1997.

UNODC. United Nations Office on Drug and Crime. World Drug Report. Viena, 2013.

VOLK, R. J.; CANTOR, S. B.; STEINBAUER, J. R.; CASS, A. R. Alcohol use disorders, consumption patterns, and health-related quality of life of primary care patients. Alcoholism Clinical and Experimental Res, v. 21, n. 5, p. 899-905, 1997. Crossef

WILSON, D.; PARSONS, J.; WAKEFIELD, M. The health-related quality-of-life of never smokers, ex-smokers, and light, moderate, and heavy smokers. Preventive Medicine, v. 29, p. 139144, 1999. crossef 